\title{
Discussion on "CFD analysis of the effect of nozzle stand-off distance on turbulent impinging jets"1
}

\author{
Rafael Duarte, Anton J. Schleiss, and António Pinheiro
}

The Discussers congratulate the Authors for their relevant work. The paper provides interesting results of CFD simulation of turbulent circular jets impinging vertically on a flat plate. Three different models were used, the realizable $k-\varepsilon$ model, the $k-\omega$ SST model, and the RSM model, and compared to experimental data from Rajaratnam et al. (2010) and Giralt et al. (1977). Many characteristics of the impinging jet were reproduced in good agreement with the experimental data.

The paper and benchmark data are based on air jets. A comparison to experimental results from an ongoing research of the Discussers on water jets may provide some important additional conclusions. The comparison made in the present discussion is based on tests with $72 \mathrm{~mm}$ diameter circular submerged water jets issued at a distance from the bottom $H / D=9.58$. Issuance velocities range from 5 to $22 \mathrm{~m} / \mathrm{s}$. More details on the experimental set up are presented by Duarte (2013) and Duarte et al. (2013).

Beltaos and Rajaratnam (1977) showed that jets impinging on a flat surface are composed of 3 distinct regions: (1) The free jet, where the jet is developing by shear with the surrounding fluid independently of the presence of the obstacle on the bottom. This region can also be divided into two sub-regions: the zone of flow development is where the core of the jet still persists and centerline velocities are thus constant, with the value of the issued jet velocity. This is followed by the zone of developed flow where the core has vanished and the centerline velocity decreases with distance from the issuance section. (2) The impingement region, created by the impact of the jet against the bottom. This results in a pressure build-up that decreases centerline velocities rapidly towards zero for a value of $x / D=H / D$, and in jet deflection parallel to the obstacle plane. (3) The wall jet region, result of jet deflection creating a flow parallel to the bottom.

Arguing that the RSM model is more accurate in the free jet region, the Authors choose to adopt this model for further investigations. However, if compared to the Discussers' data for water jets, the $k-\omega$ SST model gives a rather good agreement. Figure 1 shows the normalized centerline velocities $U_{\mathrm{c}} / U_{\mathrm{j}}$ as a function of the normalized depth below issuance $x / D$, for two jets of Reynolds number comparable to those of the Authors.

For each case, the normalized length of core decay $x_{\mathrm{c}} / D$ can be estimated as the point in the horizontal coordinate corresponding to the transition between constant and decaying velocities. It can be seen that jets with a different issuance velocity produce different lengths of core decay, which in turn are different from those obtained from Giralt et al. (1977) and from the CFD computations for air jets.
Fig. 1. Centerline velocity. Comparison of CFD results and Discussers' data for water jets.

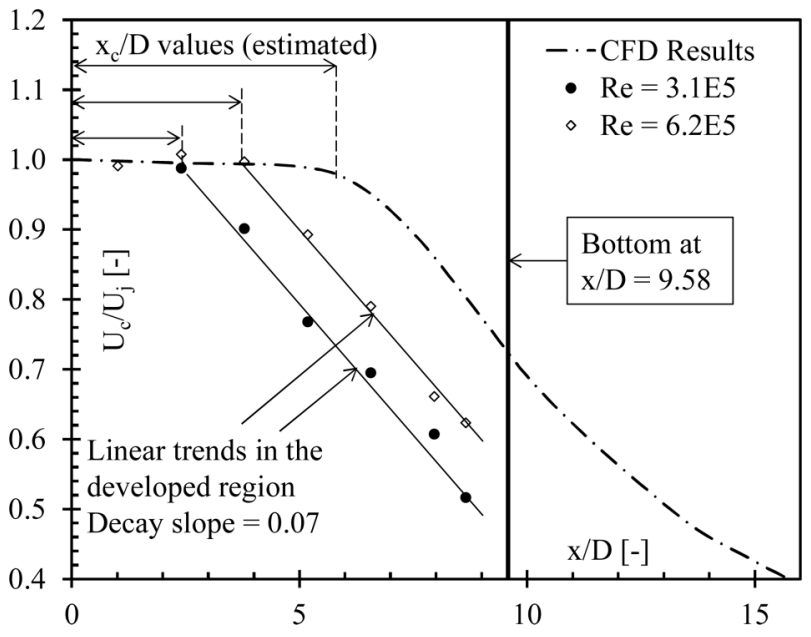

On the other hand, the decay slope in the zone of developed flow seems similar in all cases. If a linear trend is assumed in this region, a decay slope of 0.07 is found from the experimental results for water jets. This is in very good agreement with the CFD computations and with the work of Giralt et al (1977) who proposed that this slope should be 0.077 .

A closer analysis can be done if the results are plotted as a function of $x-x_{c} / D$, meaning that the zone of developed flow begins at zero in the horizontal coordinate. This is shown in Fig. 2, where experimental data for all tested water jets are shown in the same plot and compared to a linear decay and CFD results. The CFD computations fit very well the data if a $x_{c} / D$ value of 5.8 is used, very close to the Authors analysis of a core development until $x / D$ being around 6 .

As a conclusion, CFD simulations are able to accurately reproduce centerline velocities for air and water jets in the free jet region. It has to be mentioned that, according to the Authors, computations were performed with incompressible RANS equations. Also in the free jet region, the Authors call the attention to rather different behavior between the experimental data from Rajaratnam et al. (2010) and Giralt et al. (1977). They correctly suggest that this might be due to different nozzle designs that affect jet evolution. Indeed, different nozzle designs generate different turbulence intensities of the jet at the issuance section.

Received 27 November 2013. Accepted 28 November 2013.

R. Duarte and A.J. Schleiss. Laboratory of Hydraulic Constructions (LCH), Ecole Polytechnique Fédérale de Lausanne (EPFL), Station 18, CH-1015 Lausanne, Switzerland.

A. Pinheiro. CEHIDRO, Instituto Superior Técnico, Universidade de Lisboa, Av. Rovisco Pais 1049-001 Lisbon, Portugal.

Corresponding author: Rafael Duarte (e-mail: rafael.duarte@epfl.ch).

${ }^{1}$ Published in the Canadian Journal of Civil Engineering, (2013), 40: 603-612. dx.doi.org/10.1139/cjce-2012-0199. 
Fig. 2. Convergence of results for $U_{c} / U_{j}$ as a function of $x-x_{c} / D$ for air and water jets. (+) Discussers' experimental data.

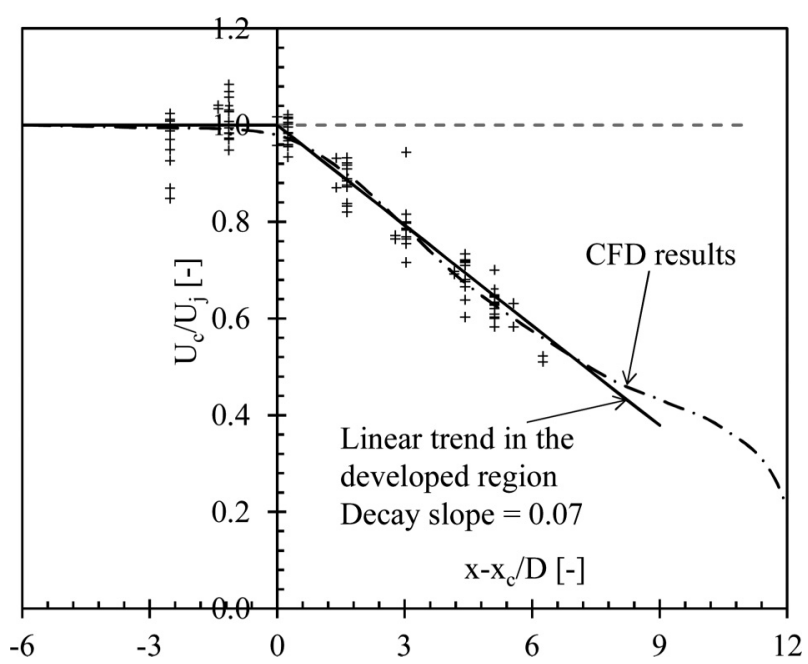

This strongly influences the formation of surface disturbances in the jet perimeter, and, as a consequence, the shear stresses with the surrounding fluid. Zhu et al. (2000) designed nozzles with the goal of minimizing surface disturbances. They were able to avoid air entrainment of plunging water jets with Reynolds numbers well above $1 \times 10^{5}$.
Nevertheless, close to the obstacle, in none of the cases was a steeper rate of velocity decay found for the water jets in the positions measured by the Discussers. For air jets, Beltaos and Rajaratnam (1977) proposed that the impingement region starts at $x_{i}=1.2 \mathrm{D}$ from the obstacle, while Giralt et al. (1977) suggested that this result is valid for nozzle heights of less than $H / D=6.8$, and for larger values: $x_{i} / D=0.153(1+H / D)$. This suggests that the impingement zone has different limits for air and water jets.

\section{References}

Beltaos, S., and Rajaratnam, N. 1977. Impingement of axisymmetric developing jets. Journal of Hydraulic Research, 15(4): 311-326. doi:10.1080/00221687709499637.

Duarte, R. 2013 Air concentrations in plunge pools due to aerated plunging high-velocity jets and dynamic pressures in underlying fissures, In Proceedings of the 35th IAHR World Congress, Chengdu, China, 8-13 September 2013. International Association for Hydro-Environment Engineering and Research, A11855, pp. 1-10.

Duarte, R., Schleiss, A.J., and Pinheiro, A. 2013 Dynamic pressure distribution around a fixed confined block impacted by plunging and aerated water jets, In Proceedings of the 35th IAHR World Congress, Chengdu, China, 8-13 September 2013. International Association for Hydro-Environment Engineering and Research, A11861, pp. 1-8.

Giralt, F., Chia, C., and Trass, O. 1977. Characterization of the impingement region in an axisymmetric turbulent jet. Industrial and Engineering Chemistry Fundamentals, 16(1): 21-28. doi:10.1021/1160061a007.

Rajaratnam, N., Zhu, D.Z., and Rai, S.P. 2010. Turbulence measurements in the impinging region of a circular jet. Canadian Journal of Civil Engineering, 37(5): 782-786. doi:10.1139/L10-014.

Zhu, Y.G., Oguz, H.N., and Prosperetti, A. 2000. Air entrainment by impinging liquid jets with surface disturbances, Journal of Fluid Mechanics, 404: 151 (2000). doi:10.1017/S0022112099007090. 